\title{
Surgical management of complicated ptotic breast
}

\author{
Opas Khomgongsuwan, MD, RCOST, RCFMT, IBCS, FTACS $®$ \\ LeBangkok Cosmetic Clinic, Bangkok, Thaliand
}

Background: Breast ptosis represents a continuous challenge for cosmetic surgeons. It is difficult to treat when patients are demanding and expect the best results with minimal scarings, low cost and short postoperative recovery time. Surgeons should know which proper surgical procedures are appropriate based on the important aspects of each case. They must choose a surgical option that helps to solve the patient's issue, meets their expectation, and results in minimal or limited complications.

Objective: Author studied the many types of breast ptosis and the surgical options for breast ptosis.

Methods: The author retrospectively analyzed 48 patients (all females; mean age, 33.7 years; age range, 22-49 years) with mild, moderate, and severe ptotic breast and different breast volumes classified by small, normal and hypertrophic breast between the 15th of January 2008 and the 30th of August 2017. The author retrospectively analyzed the type of ptotic breast, type of management and surgical result by using medical preoperative and postoperative photographs and records. Assessment of results by level of patient satisfaction is divided into poor, fair, good, and excellent. Complications are recorded each patient.

Results: Of the 48 patients of whom 45 had breast ptosis and 3 had remaining breast ptosis after previous surgery, the following surgeries were performed. Most patients had good to excellent satisfaction of results and improved the shape of their breasts. There were two cases of major complications who have superficial skin necrosis but they still resulted in good satisfaction. One patient felt she had poor improvement of the breast shape and big breasts.

Conclusion: The degree of breast ptosis and the amount of breast tissue or breast volume, are the key factors that determine surgical procedures. The selection of surgical procedure is essential and is directly related to the outcome of the surgery.

Keywords: breast augmentation; breast ptosis; crescentic mastopexy; $\mathrm{J}$ or $\mathrm{L}$ scar inverted T scar mastopexy; reduction mammoplasty; secondary mastopexy

\section{Introduction}

Breast ptosis represents a continuous challenge for cosmetic surgeons. It is difficult to treat when patients are demanding and expect the best results with minimal scarings, low cost and short postoperative recovery time. Breast ptosis can presents in many forms and can be congenital in nature, or acquired due to factors such as aging, weight changes, or pregnancy. The goal of surgery is to improve the breast shape while minimizing scarings. Many surgical procedures can manage the ptotic breast, including breast augmentation, crescentic mastopexy, vertical or short $\mathrm{T}$ scar mastopexy, reduction mammoplasty, and reduction mammaplasty with nipple areolar complex (NAC) graft [14]. Surgeons should know which proper surgical procedures are appropriate based on the important aspects of each case. They must choose a surgical options that helps to solve the patient's issue, meets their expectation, and results in minimal or limited complications. 


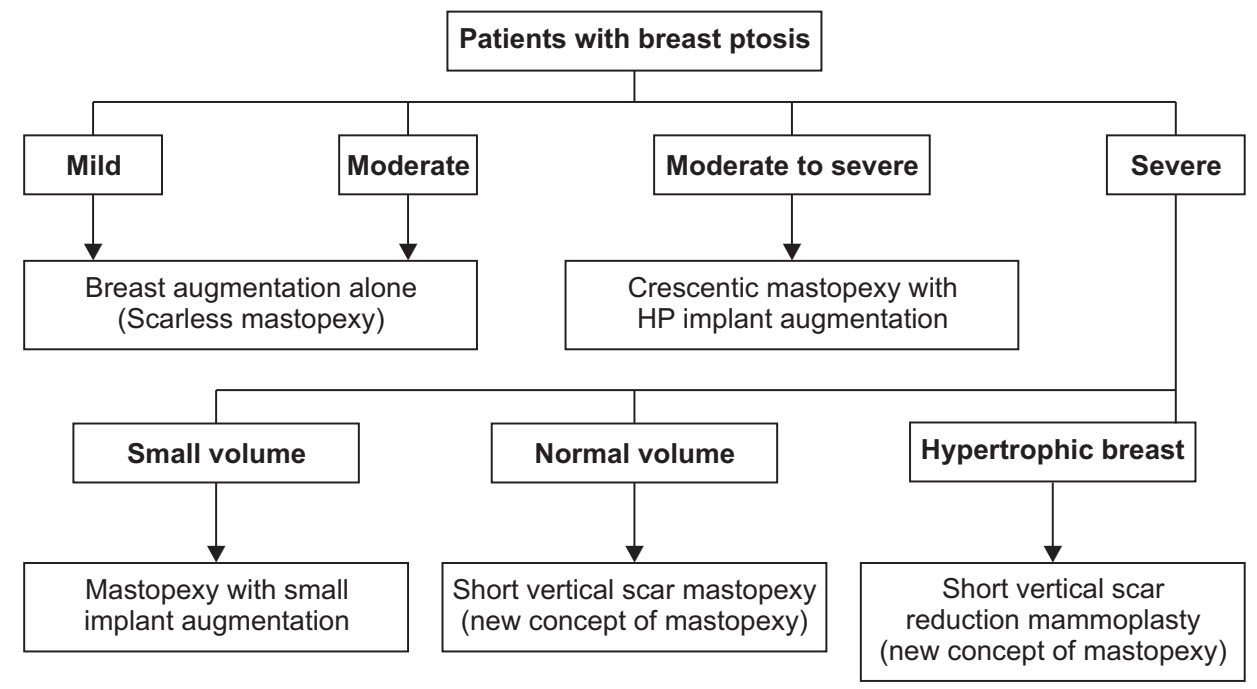

Fig. 1. Algorithm show the degree of breast ptosis and breast tissue amount or breast volume are the key factors that determine surgical procedures. HP, high profile.

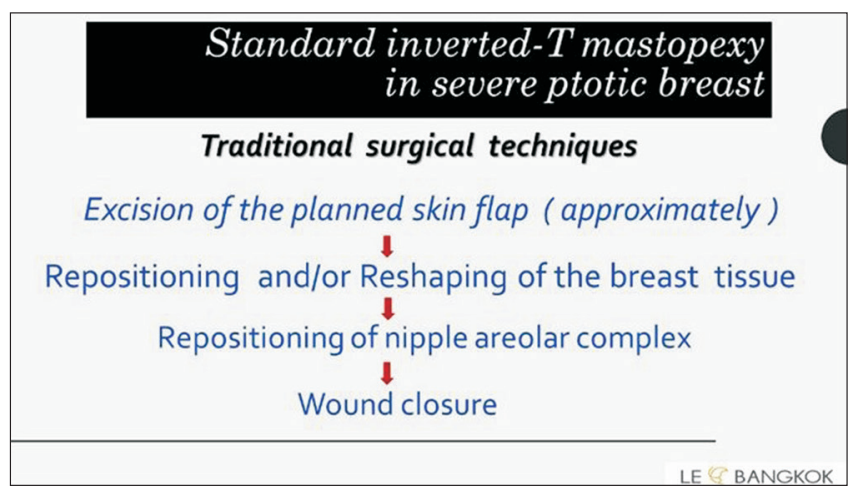

Fig. 2. Standard or traditional techniques of mastopexy make excision of the planned skin flap (approximately) before and then repositioning and/or reshaping of the breast tissue and repositioning of nipple areolar complex (NAC) finally.

\section{Materials and methods}

The author retrospectively analyzed 48 patients (all females; mean age, 33.7 years; age range, $22-49$ years) with mild, moderate, and severe ptotic breast and different breast volumes classified by small, normal, and hypertrophic breast between the 15th of January 2008 and the 30th of August 2017. The author studied the many types of breast ptosis and the surgical options chosen. The modified Regnault's classification of breast ptosis was used [5]. The degree of breast ptosis and amount of breast tissue or breast volume, are the key factors that determine the choice of surgical procedures. To study the surgical results, patient satisfaction and postoperative complications in all patients, the surgical procedures were chosen as follows, breast augmentation only or called scarless mastopexy for patients with small breast volume and no severe ptosis, breast augmentation combined

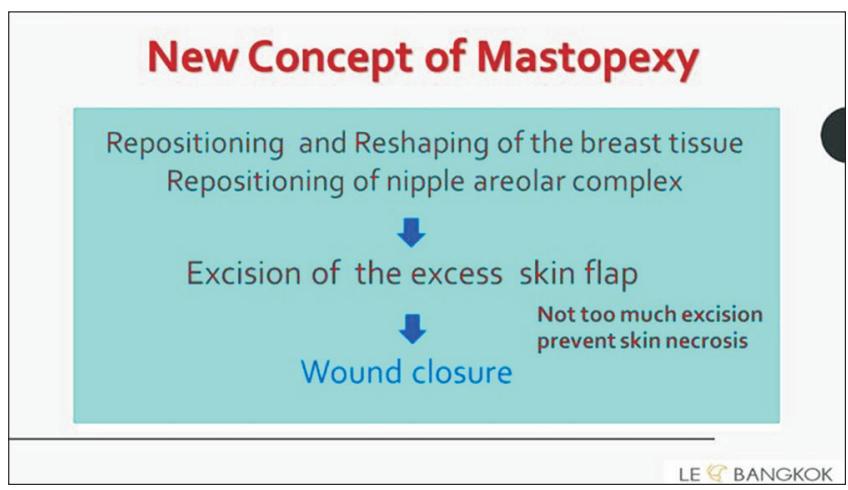

Fig. 3. New concept techniques can more precise excision of skin flap because breast tissue and nipple areolar complex (NAC) are repositioned and reshaped before. The excess skin can excise accurately after subcutaneous tissue and breast tissues remove properly and before. Excision must be enough but not too much to prevent skin closure tightly and occur skin necrosis eventually.

with crescentic mastopexy for patients with small breast volume and moderate to severe ptosis, mastopexy or reduction mammoplasty with a new concept of procedures for patients with severe ptosis (Fig. 1). The author performed the necessary procedure during tumescent anesthetic breast surgery (TABS) in an office-based surgical clinic. The management of patients with mild to moderate ptotic and small volume breast combined breast augmentation with or without crescentic mastopexy by performing primary augmentation using inframammary fold (IMF) or transaxillary approach, subglandular or dual plane I placement using larger, high profile (HP) silicone implants.

Some patients with moderate or severe ptosis cannot accept visible long visible scars. These patients are managed by combined HP implant augmentation and crescentic mastopexy. Here, patients who have severe ptosis and normal volume were 
managed by the short vertical scar mastopexy which is a new concept of procedures. New concept techniques enable the surgeon to more precise with the excision of the skin flap as breast tissue and NAC are repositioned and reshaped beforehand while the standard or traditional techniques of mastopexy make the excision of the planned skin flap (approximately) before and then reposition and/or reshape the breast tissue and reposition NAC at the end of the procedure [1-4]. The excess skin can be excised accurately after subcutaneous tissue and breast tissues removed properly and completely. The excised skin must be ample but not too much to prevent tight skin closure and to ensure skin necrosis do not occur. (Fig. 2, 3). Patients who have
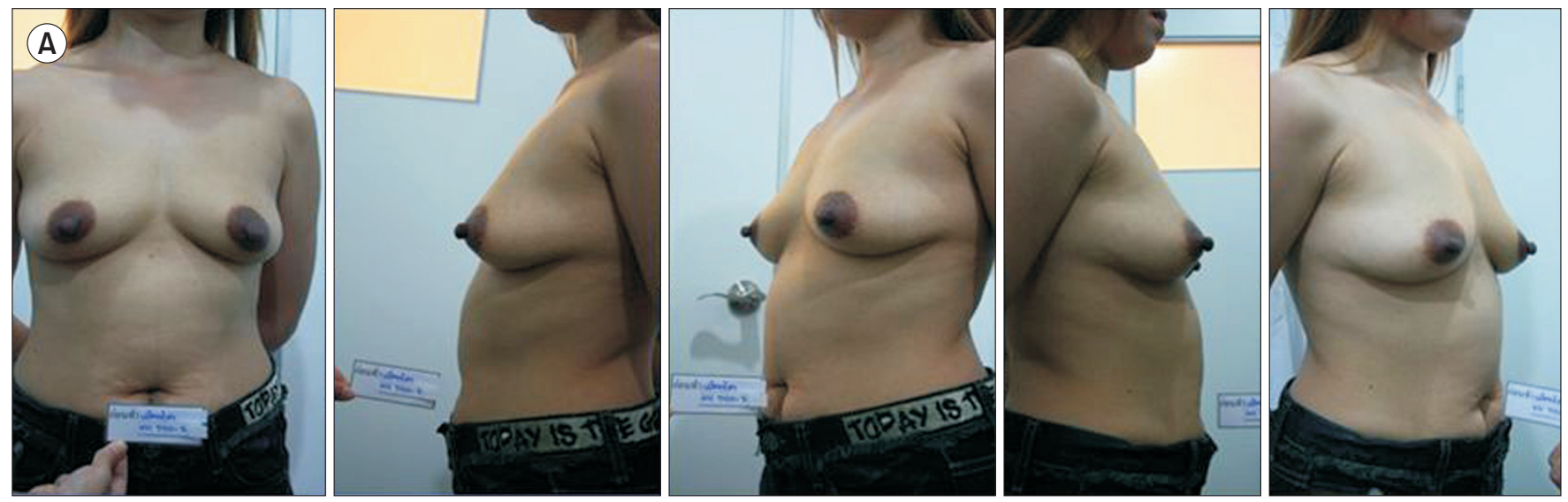

Preoperative
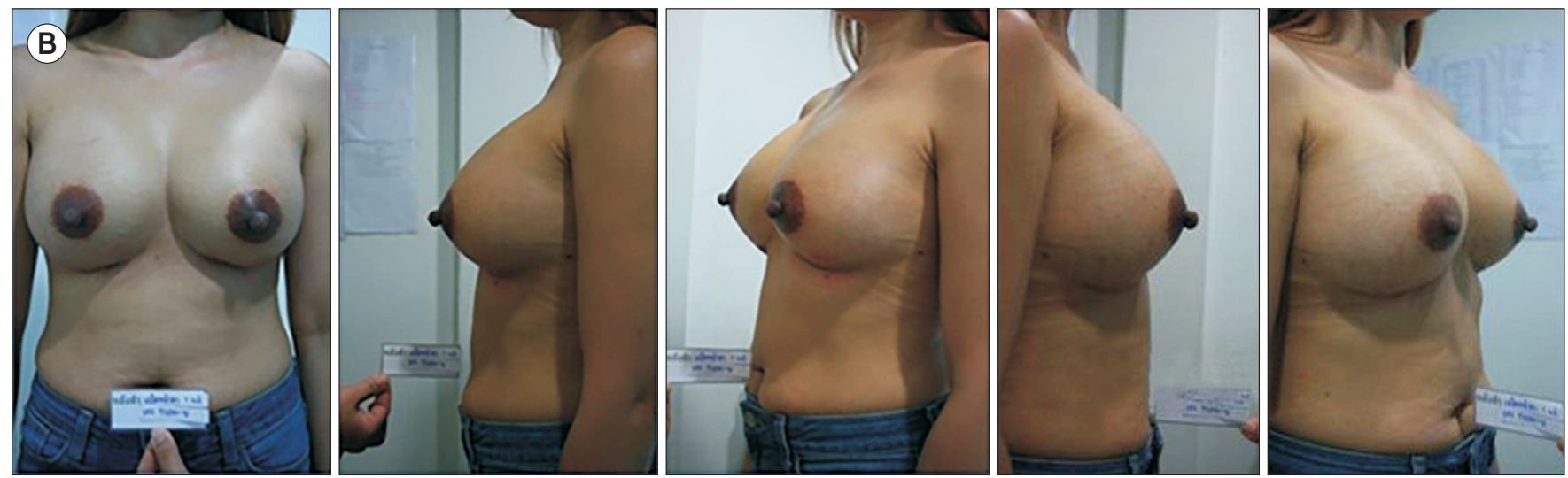

$340 \mathrm{cc}, \mathrm{MHP}$ allergan, subglandular plane, postoperative $1 \mathrm{wk}$
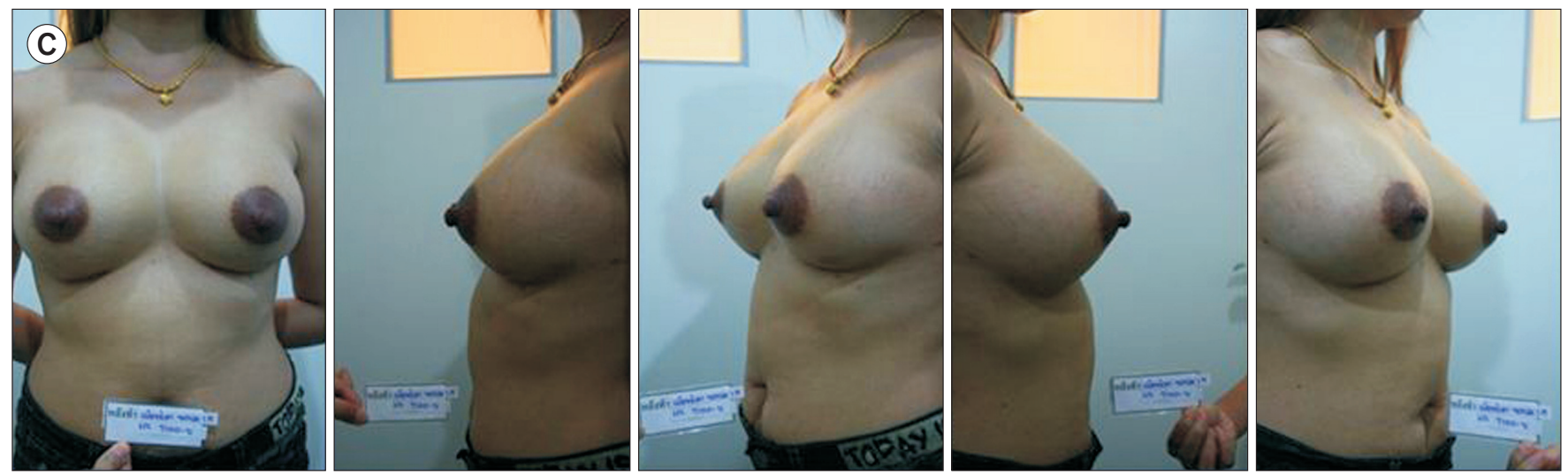

Postoperative 1 mo

Fig. 4. Patient with mild to moderate ptosis and small volume is managed by the high profile (HP) breast augmentation. This case use 340 cc of moderate high profile (MHP) implant subglandularly. Pictures show preoperatively (A), one week (B) and one month (C) postoperatively. 
severe ptosis and small or large hypertrophic breasts are treated using the same new concepts in a vertical or short T-inverted scar reduction mammoplasty. For secondary ptotic breasts, patients were managed by same manner. Redivac drains are placed postoperatively and removed around 1 or 2 days after surgery in each case. The type of ptotic breast, type of management and surgical result were analyzed retrospectively using medical preoperative and postoperative photographs and records. Assessment of results by the level of patient's satisfaction was divided into poor, fair, good, and excellent. Complications were recorded each in patient.

\section{Results}

Of the 48 patients, of whom 45 had breast ptosis and 3 had remaining breast ptosis after previous surgery, the following surgeries were performed, the inframammary approach for breast augmentation only (Fig. 4-6), the upper half periareolar approach for crescentic mastopexy (Fig. 7, 8), or short vertical scar for mastopexy and reduction mammoplasty with new concept procedure (Fig. 9, 10). Most patients had good to excellent satisfaction of the results and improved the shape of their breasts. There were two cases of major complications but they still resulted in good satisfaction. One patient who underwent reduction mammoplasty, had superficial skin necrosis of right breast which was located at the junction of the inferior edge of areola and vertical incision. Another patient, who underwent secondary mastopexy, had superficial skin necrosis of the same area of both sides. Skin necrosis was managed by, removal of the death tissue, and redressing the wet wound every day to ensure delayed primary wound closure. One patient felt she had poor improvement of breast shape and big breasts. She had moderate to severe ptotic breasts and implied she did not want a vertical scar on her breast. Her selection was the crescentic mastopexy with a higher size and HP implant augmentation and the patient required mastopexy with small implant aug-
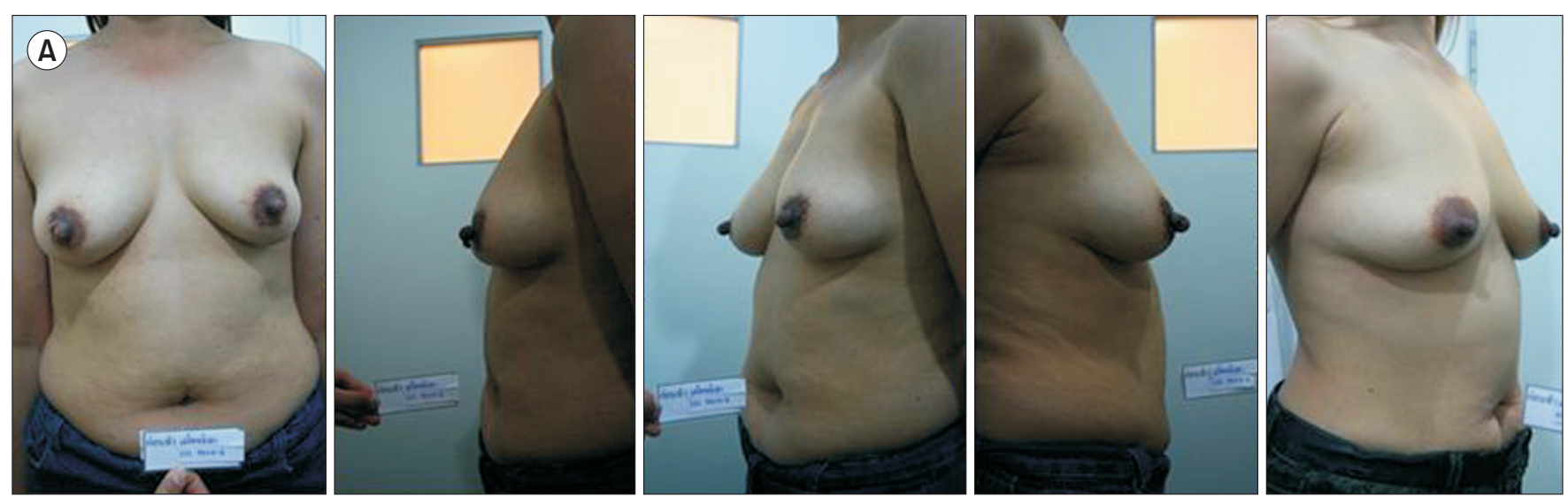

Preoperative
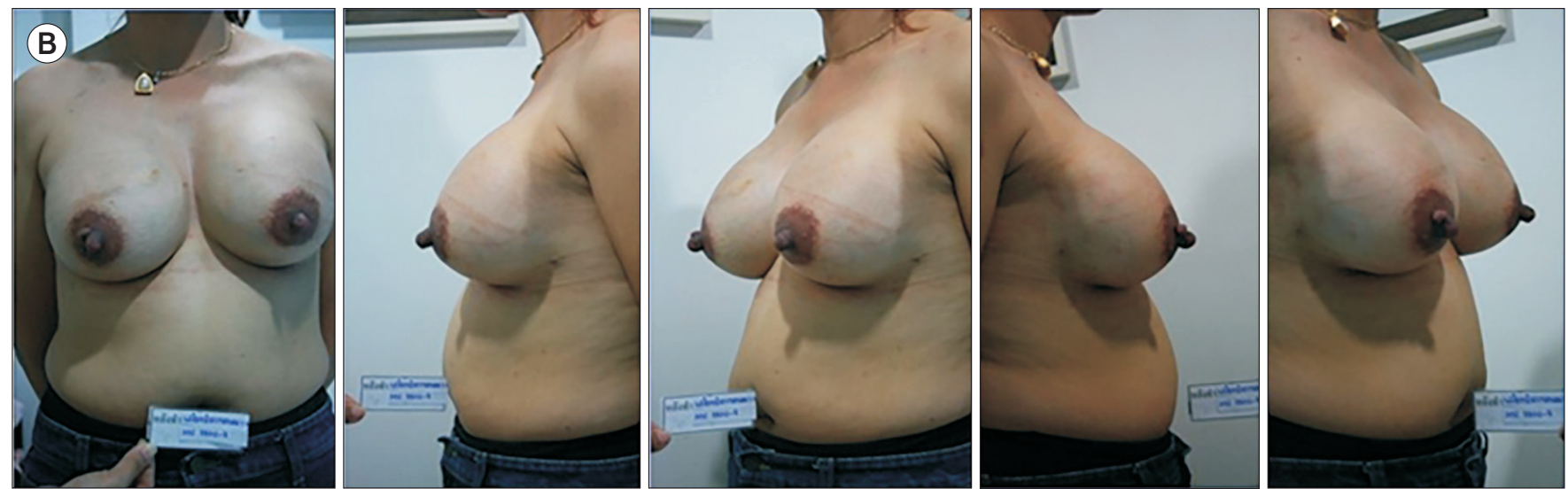

$380 \mathrm{cc}, \mathrm{MHP}$ allergan, subglandular plane, postoperative $1 \mathrm{wk}$

Fig. 5. Patient with mild to moderate ptosis and small volume is managed by the high profile (HP) breast augmentation. This case use 380 cc of moderate high profile (MHP) implant subglandularly. Pictures show preoperatively and one-week postoperatively. 

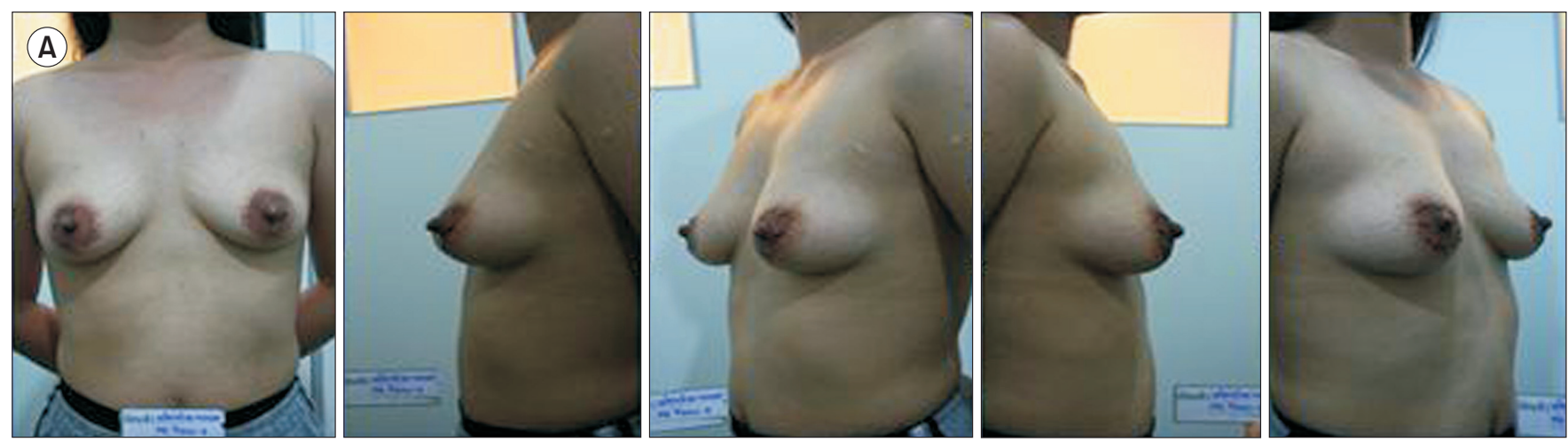

Preoperative
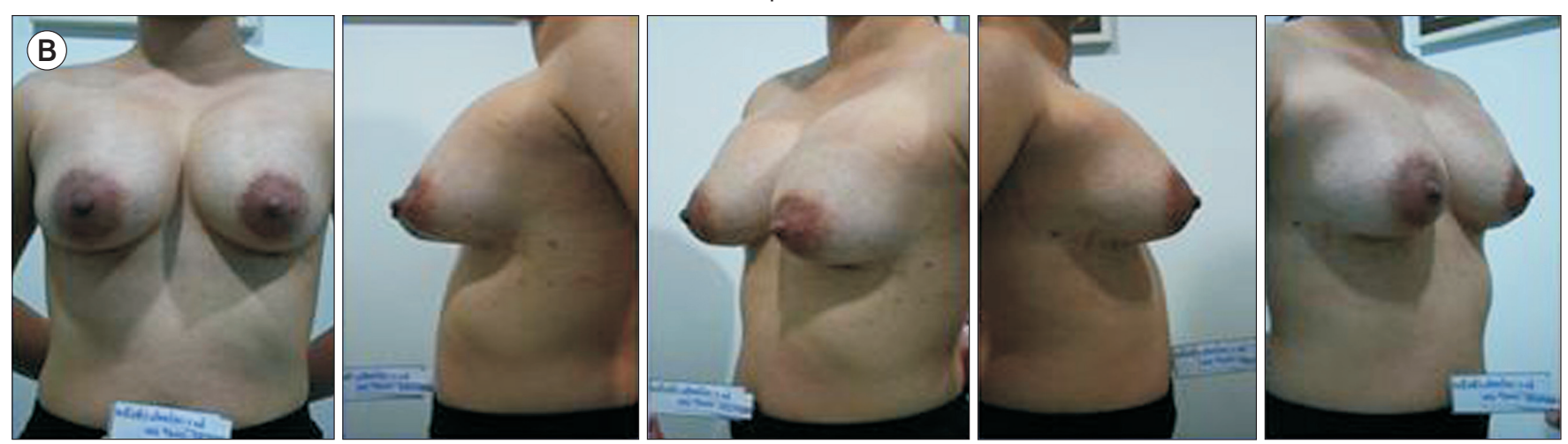

$325 \mathrm{cc}, \mathrm{HP}$ silimed, dual plane I, postoperative $1 \mathrm{wk}$
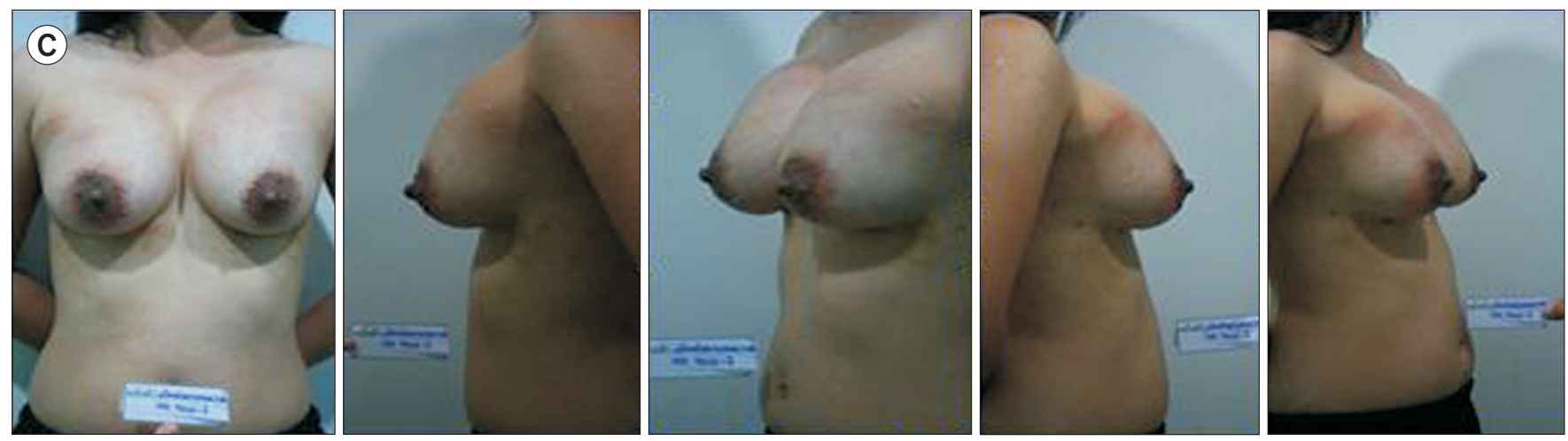

Postoperative 2 wk
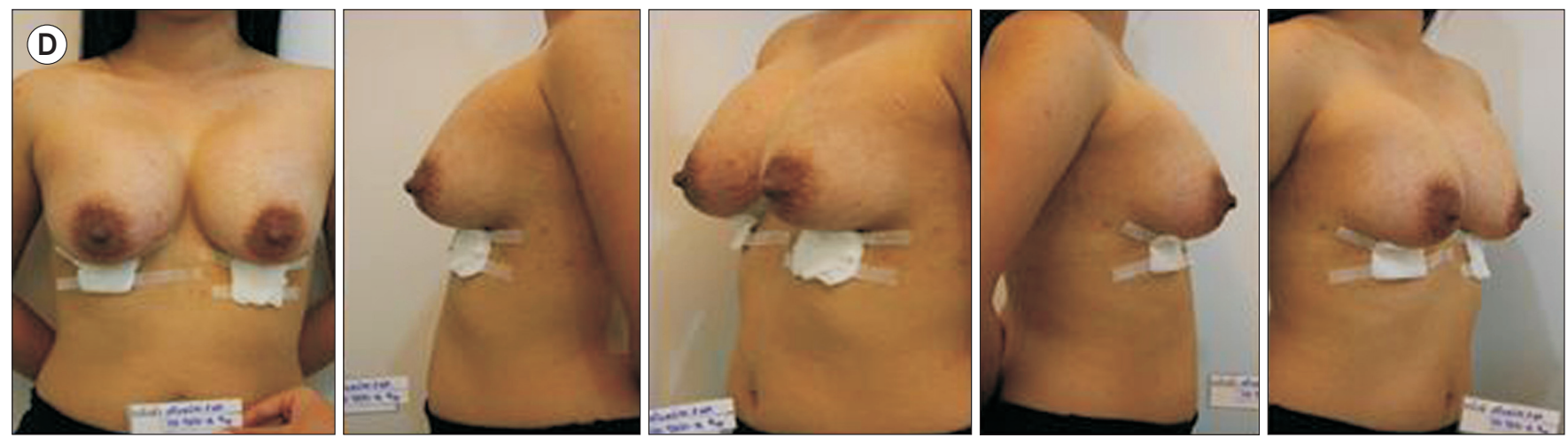

Postoperative $3 \mathrm{wk}$

Fig. 6. Patient with mild to moderate ptosis, small volume and thin upper pole is managed by the high profile (HP) breast augmentation subpectorally. This case use $325 \mathrm{cc}$ of high profile implant. Pictures show preoperatively (A), one-week (B), two-week (C), and three-week postoperatively (D). She has the postoperative snail-shell deformity. Deformity is temporary and will improve gradually. 

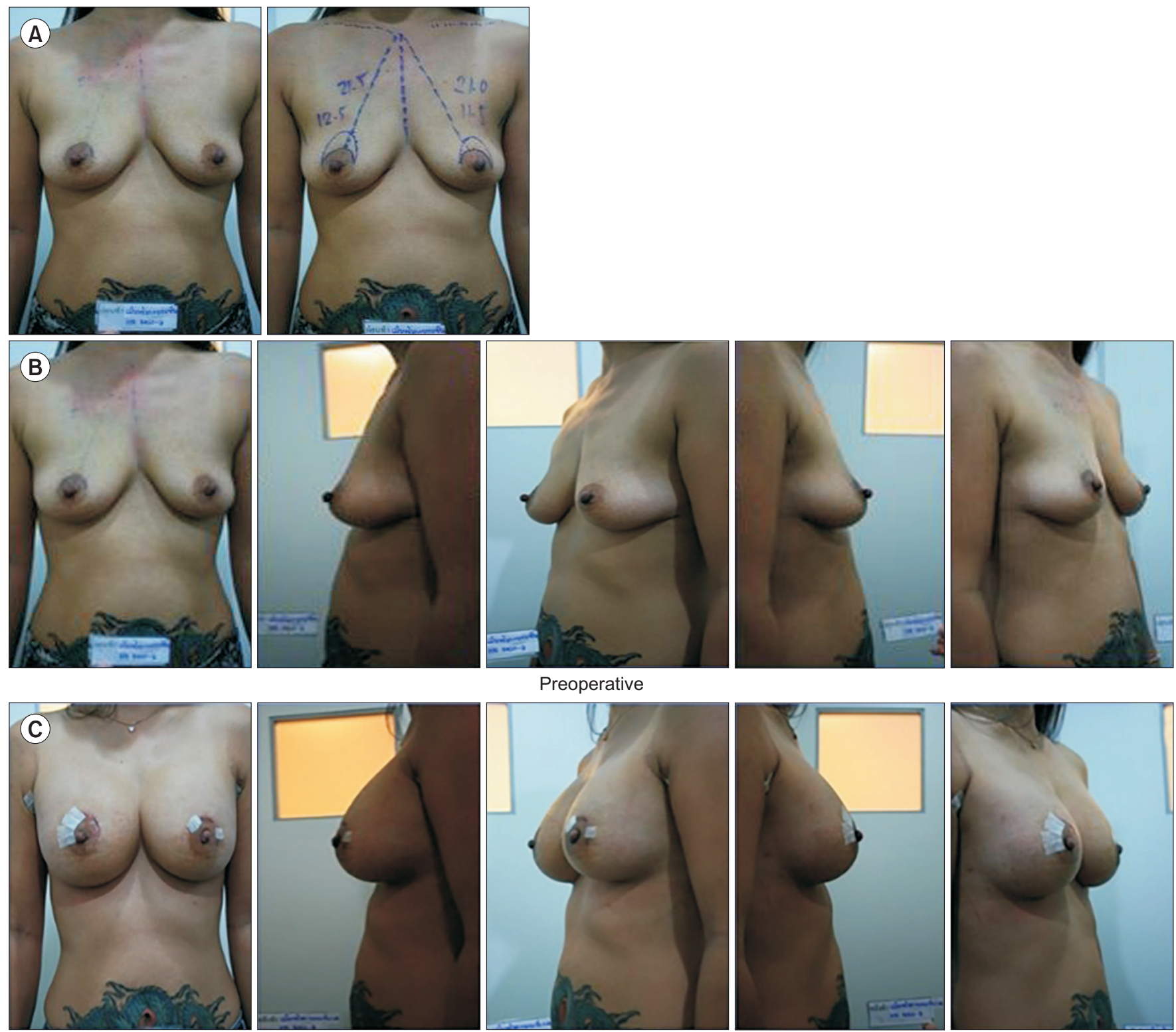

$350 \mathrm{cc}, \mathrm{HP}$ CUI allergan, subpectoral plane, postoperative 2 wk
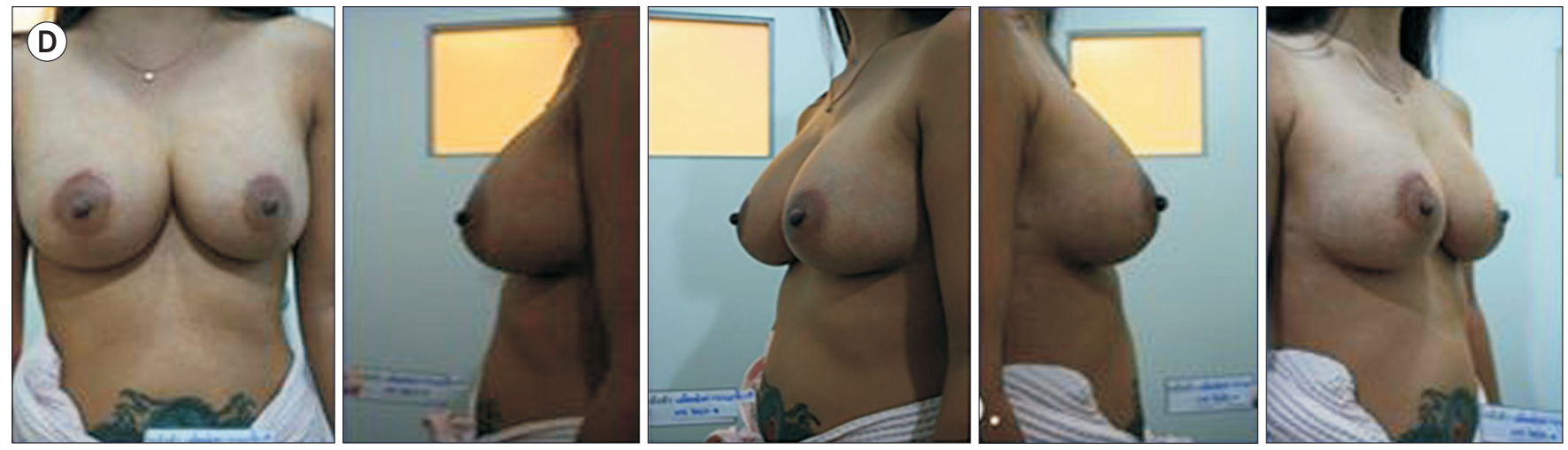

Postoperative $1 \mathrm{mo}$

Fig. 7. Case I. (A) Preoperative planning of crescentic mastopexy which upper half periareolar approach in patients with thin upper pole, moderate to severe ptosis and small breast volume. (B) Preoperative pictures. Two-week (C) and one-month (D) postoperative pictures of patients managed crescentic mastopexy and subpectorally $350 \mathrm{cc}$ high profile (HP) breast augmentation. 

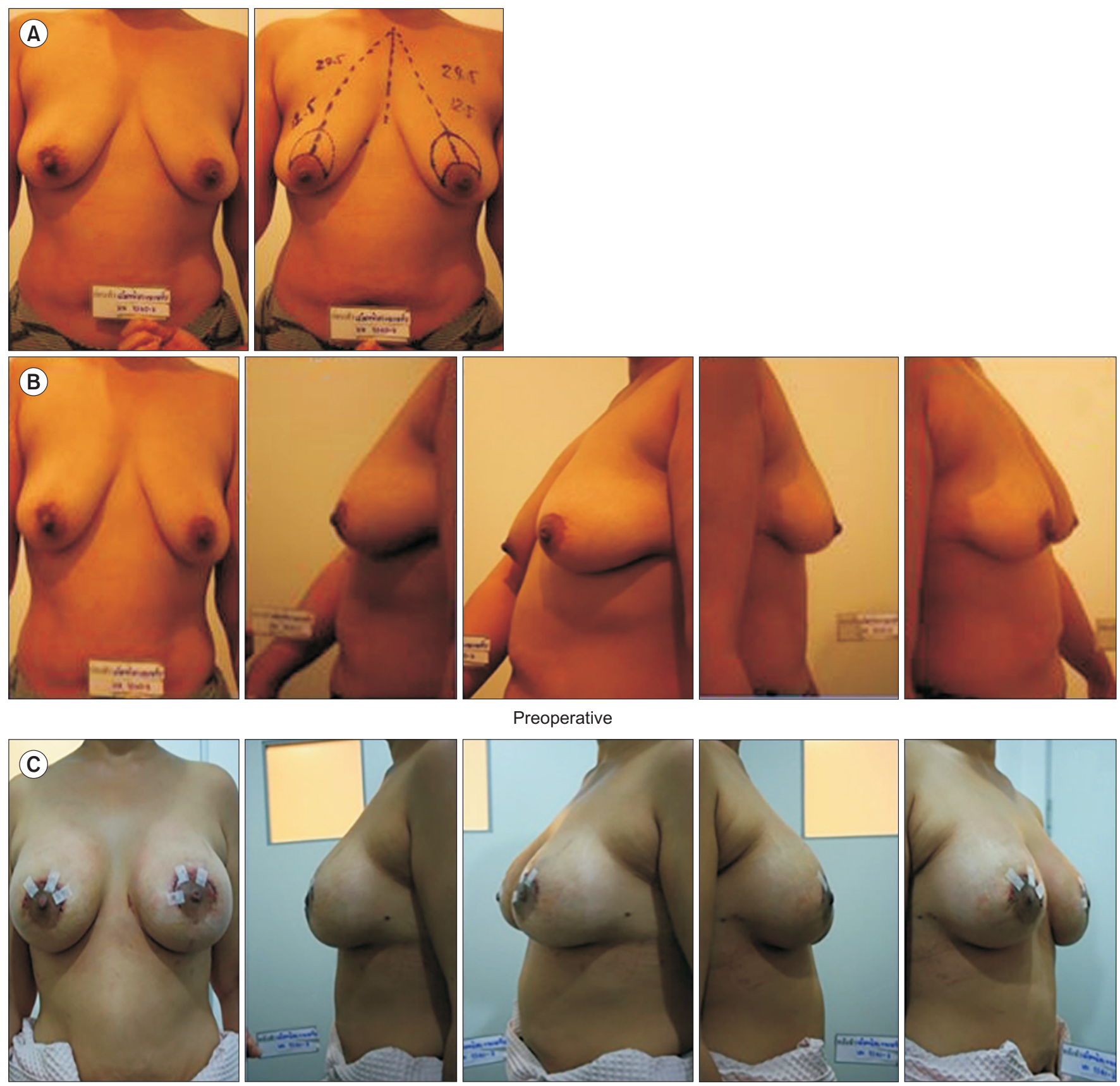

$325 \mathrm{cc}, \mathrm{HP}$ quartzo silimed, subglancular plane, postoperative $2 \mathrm{wk}$

Fig. 8. Case II. (A) Preoperative planning of crescentic mastopexy which upper half periareolar approach in patients with moderate to severe ptosis and small breast volume. (B) Preoperative pictures. (C) Two weeks postoperative pictures of patients managed crescentic mastopexy and subgrandularly $325 \mathrm{cc}$ high profile (HP) breast augmentation.

mentation later.

A snail shell deformity complication occurred in a patient who had mild to moderate ptosis and was treated by subpectorally HP breast augmentation (Fig. 11) [6]. In the immediate postoperative period, the patient has an exaggerated superior implant displacement due to the compressive forces of the pec- toralis and lateral resisting forces of the serratus muscles when the implant was placed in a complete or modified dual plane I. This "snail-shell" appearance, although temporary lasting over a 6 to 12 weeks period, is more exaggerated in implant sizes greater than $350 \mathrm{cc}$ and when the level of ptosis is more severe [6]. Another complication encountered with crescentic masto- 

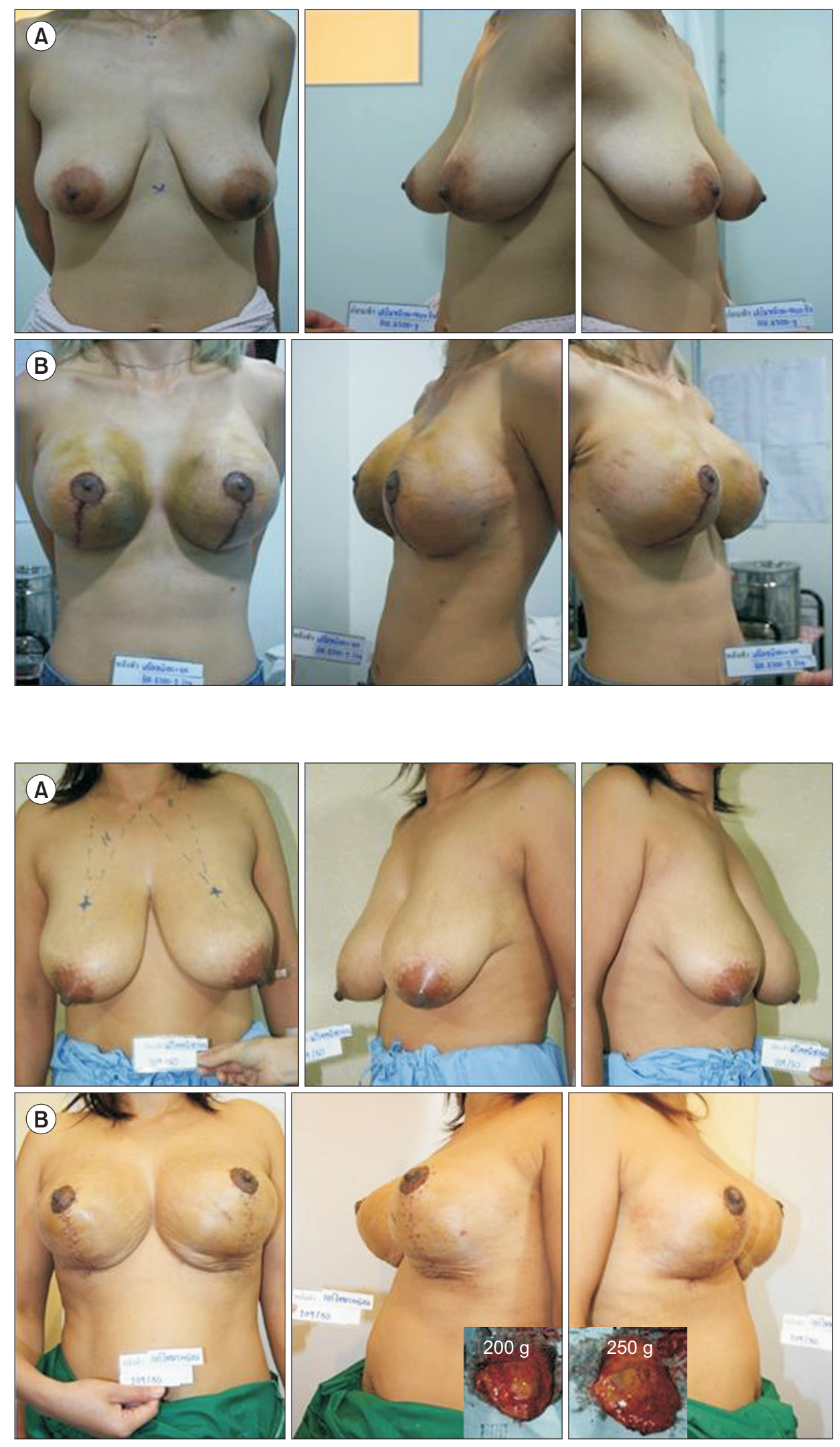

Fig. 9. Patient with severe ptosis, and small volume is managed by mastopexy with small breast implant augmentation. This case use 325 cc of high profile implant. Pictures show preoperatively (A), sixteenth day postoperatively (B). Both breasts has postoperative bruise. Bruise is temporary and complete recovery within three to four weeks.

Fig. 10. Patient with severe ptosis, and hypertrophic breast is managed by reduction mammaplasty with vertical scar only. This case remove the $200 \mathrm{~g}$ and 250 $\mathrm{g}$ of excess breast tissue from right and left breast respectively. Pictures show preoperatively (A), seventeenth day postoperatively (B). 
pexy is the widening of the periareolar scar and/or hypertrophic scar. This was prevented by a bra lift below the breast after surgery for at least 3 to 6 months to reduce the wound tension

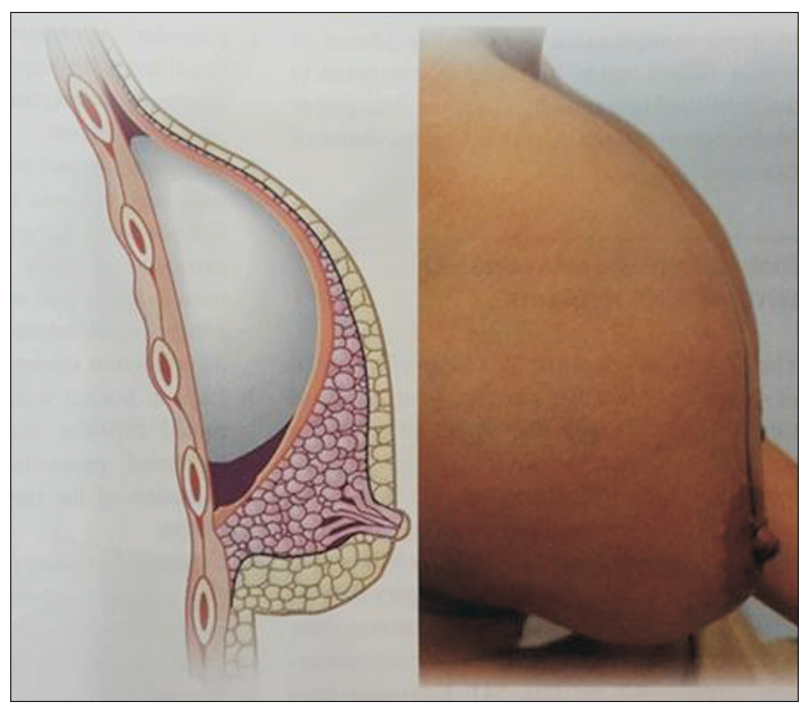

Fig. 11. This picture show "snail-shell" appearance, although temporary over 6 to 12 weeks period, is more exaggerated in implant sizes greater than $350 \mathrm{cc}$ and when the level ptosis is more severe. Cited from Novoa JC (The use of high-profile and extra-high-profile implants in ptotic breasts as an alternative to staged augmentation/mastopexy. In: Mugea TT, Shiffman MA, editors. Aesthetic surgery of the breast; 2015. p. 757) [6]. during healing and to prevent obvious scars. Other complications, including nipple sensations are relatively normal. Some patients have decreased sensation and this fully recovers within three months. Some patients have greater amount of bruising in cases of mastopexy or reduction mammoplasty (Table 1).

\section{Discussion}

Patients with a small breast volume and no severe ptosis are appropriate candidates for breast augmentation. Breast augmentation alone is a good choice for these patients but is not always appropriate if the surgeon performs this operation in patients with severe ptotic breasts. Breast augmentation can correct the aesthetics for patients with mild to moderate ptosis. The

Table 1. Postoperative complications

\begin{tabular}{|lc|}
\hline \multicolumn{1}{|c}{ Complications } & No. of cases (total=48) \\
\hline Skin bruise & All \\
\hline Superficial skin necrosis & 2 \\
\hline Patient unsatisfaction & 1 \\
\hline Widening of wound & 0 \\
\hline Hypertrophic scar & 0 \\
\hline Temporal numbness of NAC & Fully recovery within 3 mo \\
\hline
\end{tabular}

NAC, nipple areolar complex.

Hx: severe ptosis operated with breast augmentation
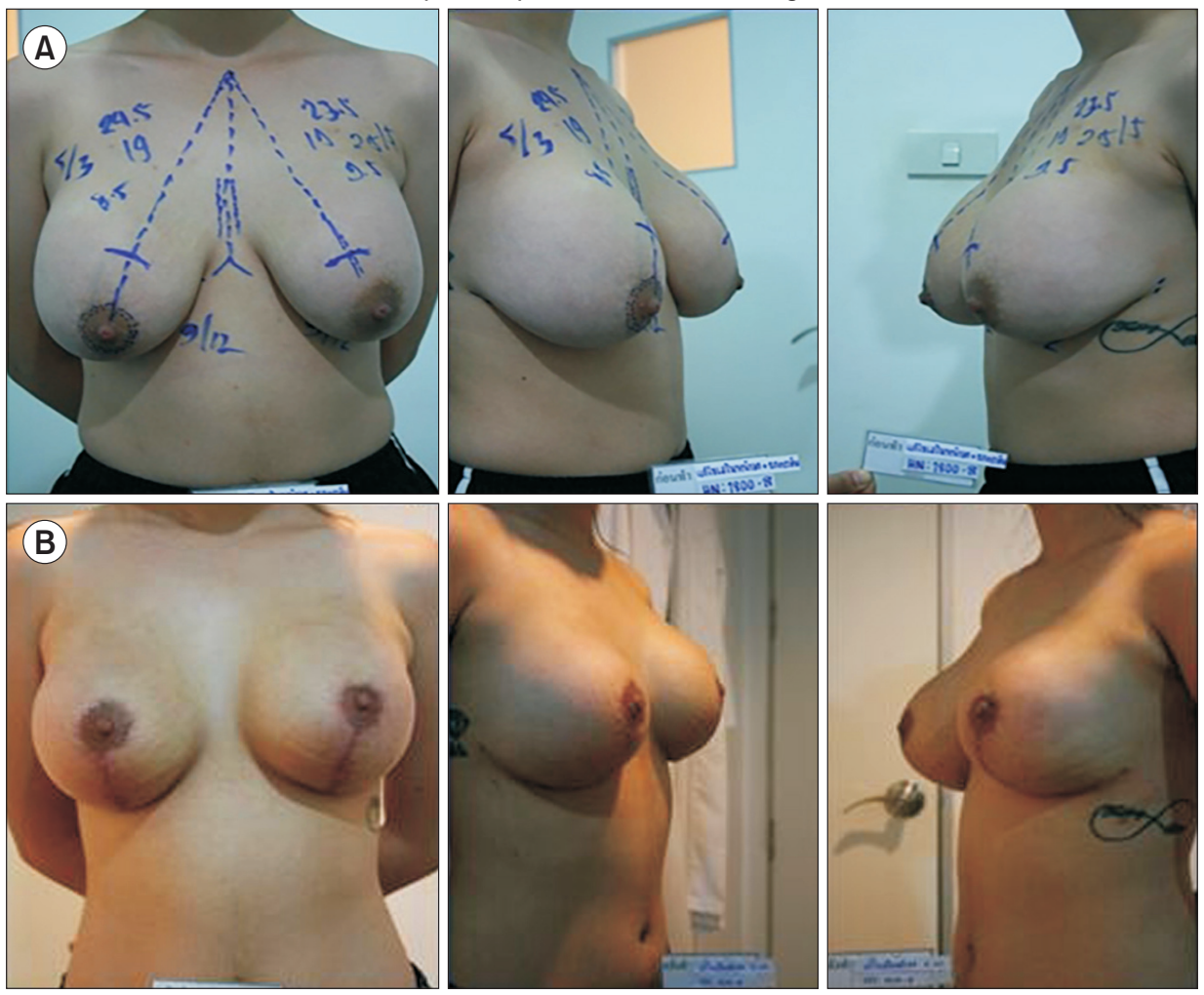

Fig. 12. Case I. Patient with severe ptosis, and normal breast volume is operated previously from others by breast augmentation. This case manage by mastopexy and secondary breast augmentation with small implant. Pictures show preoperatively (A), five weeks postoperatively (B). Hx, history. 
projection and size of implants are the most important factors to correct breast ptosis. HP implants and the one or two more estimated size of breast implant with soft tissue concept, should be chosen to receive satisfactory results. Although HP implants are an acceptable choice for primary breast augmentation, and are ideal for glandular mild to moderate ptosis, due to the higher projection forces of HP implants, the risk of excessive atrophy, compromised tissue vascularity, and excessive tissue thinning should be considered when placing larger and/or HP implants in a subglandular plane.

In cases of severe ptotic breasts, many surgical techniques of mastopexy should be chosen only if patients have normal breast volume and reduction mammoplasty is appropriate for hypertrophic breasts. Standard mastopexy procedures excise the planned skin flap approximately before repositioning of the glandular tissue and NAC, therefore the main complications are skin excess, asymmetry, not perfectly shaped breast, long scar patterns including circumvertical (including J or L scar variations), and inverted-T patterns. Here a new concept of mastopexy was presented with excellent results. The concept consists of alterations in the steps of the procedure which performs repositioning and reshaping of the glandular tissue and repositioning of NAC followed by design and excision of the skin flaps to increase accuracy. Postoperative asymmetry and long undesired scarring are resolved with this new technique. There has yet to be an additional reshaping procedure of glandular tissue performed to obtained a beautiful lower pole of the breasts. Reshaping using an atraumatic, long curved clamp makes the convex shape of lower pole of the breast. J or L scars or inverted-T patterns can be decreased into short vertical scars only. Likewise, this concept can be used to reduce the common complications of reduction mammoplasty, such as postoperative asymmetry or, improper flap excision in addition to reducing the size of scarring. Mastopexy and reduction mammoplasty were performed for two patients who had superficial skin necrosis at the areolar-vertical incision. Skin necrosis is caused by removing too much excess skin which makes the stitching too tight and is common in patients who underwent this step of surgery at the beginning of the operation or in patients who smoke excessively, this complication is discussed with patients
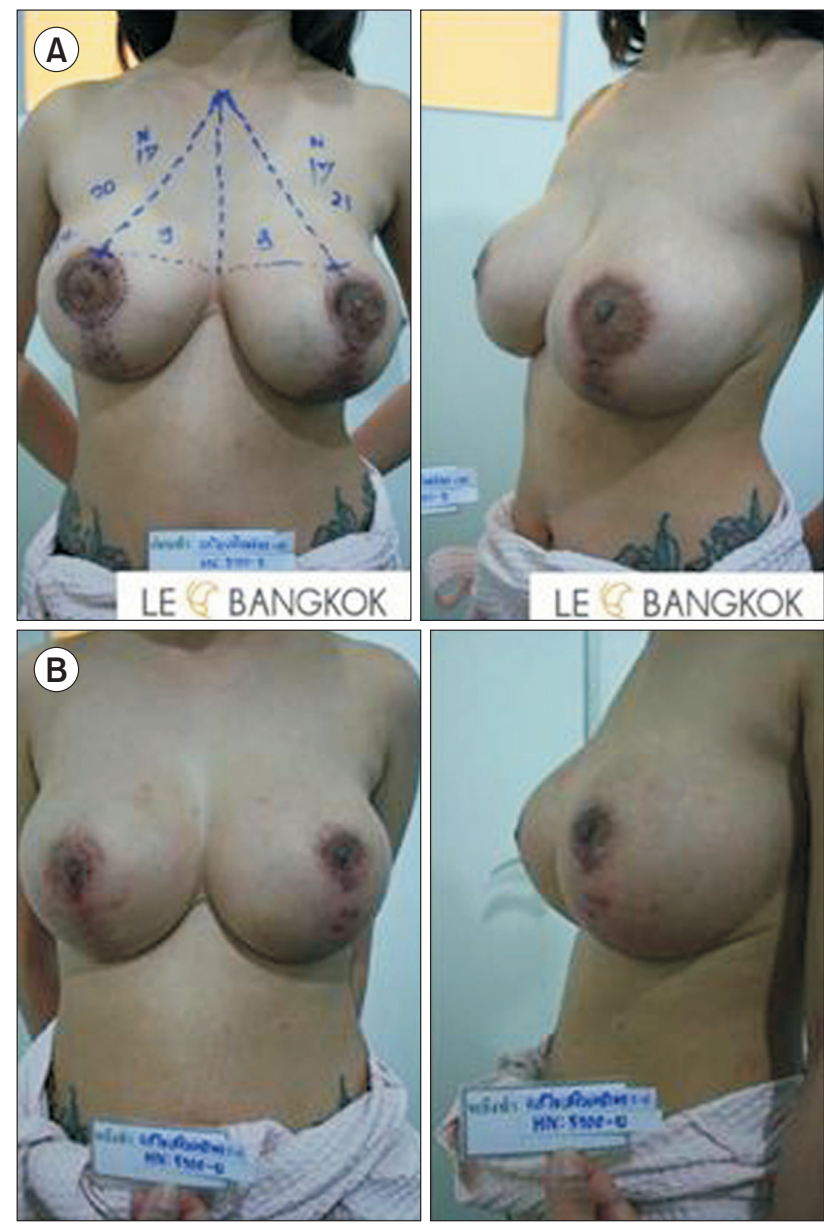
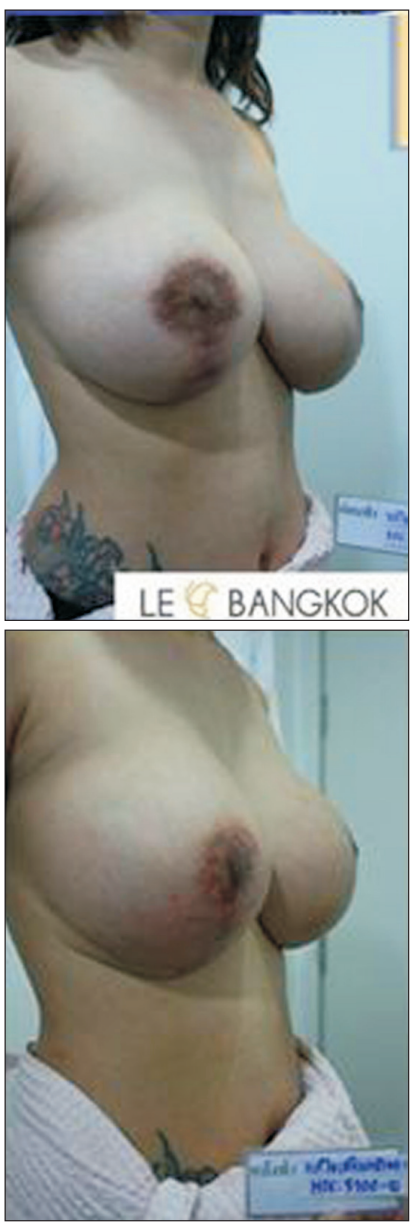

Fig. 13. Case II. Patient with previous second mastopexy with breast augmentation. Her problems are multioperated, widening of periareolar and vertical scar, capsular contracture grade II and unequal breast. This case manage by scar excision, areolar reduction, capsulotomy and partial capsulectomy, third mastopexy and breast augmentatiom. Pictures show preoperative markings, unequal breast, widening of scar both sides (A) and two months postoperatively (B). 


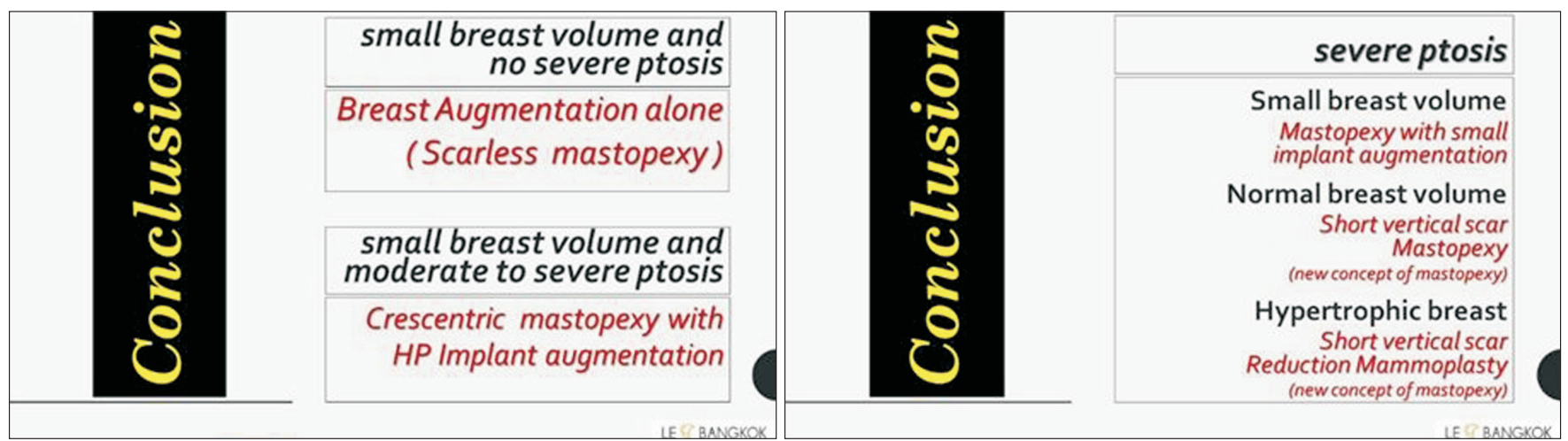

Fig. 14. Management options depend on severity of ptotic breast and breast volume.

preoperatively.

This study shows, it is possible to combine crescentic mastopexy with HP implant augmentation in patients who have moderate to severe ptotic and small volume breast. These combined procedures help to improve ptosis and shape of breast especially patients with severe ptosis who would rather avoid a long scar like an inverted $\mathrm{T}$ scar or even short vertical scar. Patients can have satisfactory breast shape and ptotic improvement but there are reports of widening of periareolar scar and/ or hypertrophic scar. It is believed this complication comes from patients not wearing a support bra according to the doctor's recommendations and the bra not lifting below the breast after surgery for at least 3 to 6 months.

For secondary ptotic breasts, this was managed depending on the problem of each breast. Common management includes capsulotomy, partial capsulectomy and changing of the pocket of breast implant into the dual subpectoral plane I in capsular contracture, changing of the more size and/or higher profile of implant in more ptosis patients, changing of the more size and/ or higher profile of implant of one side and mastopexy of another side in unequal ptotic breast, or a new mastopexy on both sides in patients with remaining severe ptosis after previous surgery (Fig. 12, 13) [7].

In conclusion, degree of breast ptosis and breast tissue amount or breast volume are the key factors that determine surgical procedures. Selection of surgical procedure is important and is directly related to the results of the surgery that has been received. Cases with small volume and no severe ptotic breast are managed by breast augmentation alone (scarless mastopexy), ones with small volume and moderate to severe ptotic breast should be treated with crescentic mastopexy with HP augmentation, and severe ptotic breast are managed by masto- pexy with small implant for small breast volume, short vertical scar mastopexy in new concept for normal breast volume, and short vertical scar reduction mammaplasty using the new concept for hypertrophic breast (Fig. 14). It is important to select the correct surgical procedure.

\section{Conflicts of interest}

The author has nothing to disclose.

\section{References}

1. Nahabedian MY, Neligan PC. Plastic surgery. 4th ed. Amsterdam: Elsevier; 2017.

2. Grotting JC, Marx AP, Chen SM. Mastopexy. In: Mathes SJ, Hentz VR, editors. Plastic surgery: tumors of the head, neck and skin. 2nd ed. Philadelphia: Elsevier; 2006. p. 47-86.

3. Mugea TT. Breast reduction/mastopexy with short invertedT scar. In: Mugea TT, Shiffman MA, editors. Aesthetic surgery of the breast. Berlin: Springer; 2015. p. 767-96.

4. Spear SL, Newman MK. Mastopexy with and without implant. In: Aston SJ, Steinbrech DS, Walden J, editors. Aesthetic plastic surgery. Edinburg: Saunders; 2009. p. 601-8.

5. Mugea TT. Breast augmentation and mastopexy. In: Mugea TT, Shiffman MA, editors. Aesthetic surgery of the breast. Berlin: Springer; 2015. p. 693-721.

6. Novoa JC. The use of high-profile and extra-high-profile implants in ptotic breasts as an alternative to staged augmentation/mastopexy. In: Mugea TT, Shiffman MA, editors. Aesthetic surgery of the breast. Berlin: Springer; 2015. p. 739-63.

7. Shestak KC. Reoperative plastic surgery of the breast. Philadelphia: Lippincott Williams \& Wilkins; 2006. 